\title{
Evolution of genetic diversity in metapopulations: Arabidopsis thaliana as an experimental model
}

\author{
Claire Lavigne*a, Xavier Reboud ${ }^{\mathrm{b}}$, Madeleine LeFranC ${ }^{\mathrm{a}}$, \\ Emmanuelle PORCHER ${ }^{\mathrm{a}}$, Fabrice ROUX ${ }^{\mathrm{b}}$, Isabelle OLIVIERI ${ }^{\mathrm{c}}$, \\ Bernard GODELLE $^{\mathrm{d}}$
}

a Laboratoire écologie, systématique et évolution, Université Paris-XI/Cnrs UPRES-A 8079, bâtiment 362, 91405 Orsay Cedex, France

${ }^{\mathrm{b}}$ Laboratoire malherbologie et agronomie, Institut national de la recherche agronomique, BP 86510, 21065 Dijon Cedex, France

${ }^{c}$ Institut des sciences de l'évolution, Université Montpellier 2,

Place Eugène Bataillon, 34095 Montpellier Cedex 05, France

${ }^{\mathrm{d}}$ Laboratoire génome, populations, interactions, Université de Montpellier II, case courrier 063, bâtiment 13, 34095 Montpellier Cedex 05, France

\begin{abstract}
Two experiments were set up to investigate how to maintain or create genetic diversity in artificial or managed populations of plants. Using Arabidopsis thaliana, we established 18 metapopulations of 20 populations each, all with the same initial genetic composition. We tested the effects of the population size, the artificial selection regime and the extinction/recolonisation regime. We report the results of the first four generations of evolution for a trait under selection (precocity) and for allozyme diversity. As expected, overall diversity decreased in each metapopulation, and differentiation among populations increased. As expected, the differentiation was weaker for larger population sizes and in the treatment with extinction and recolonisation with no bottleneck. Artificial selection was effective because the life cycle duration was much reduced. However, most of the reduction occurred during the first generation. We observed an increase of one allele at the LAP-2 locus in all metapopulations, breaching neutral assumptions for this locus. Finally, the selection regime made little difference for small population sizes, whereas large metapopulations were more differentiated when artificial selection was heterogeneous among populations. Altogether, our results agree with theoretical expectations, and provide some new results, which could not have been anticipated. In particular, the overall decrease in genetic diversity was very large (of the order of $20 \%$ in 4 generations) even for metapopulations of 2000 individuals.
\end{abstract}

genetic diversity / experimental evolution / conservation / small populations / precocity

\footnotetext{
* Correspondence and reprints

E-mail: claire.lavigne@esv.u-psud.fr
} 
Résumé - Évolution de la diversité génétique en métapopulation : Arabidopsis thaliana comme modèle expérimental. Deux expérimentations, visant à étudier comment maintenir ou créer de la diversité dans les populations artificielles ou gérées de plantes, ont été menées en utilisant Arabidopsis thaliana comme espèce modèle. Nous avons créé 18 métapopulations de 20 populations de même composition initiale. Nous avons testé les effets de la taille des populations, du régime de sélection et du régime d'extinction/recolonisation. Les résultats sur l'évolution du caractère sélectionné (durée du cycle de vie) et sur la diversité enzymatique sont présentés pour les quatre premières générations. Conformément aux attendus théoriques, la diversité a globalement diminué dans les métapopulations et les populations se sont différenciées. La différenciation est plus faible pour les grandes populations et dans le traitement avec extinctions et recolonisations sur une base génétique large. La sélection artificielle a réduit la durée du cycle des plantes. Cette réduction a essentiellement eu lieu pendant la première génération. Nous avons observé une augmentation d'un des allèles au locus LAP-2 dans toutes les métapopulations, en contradiction avec la neutralité présupposée de ce locus. Enfin, la sélection a eu peu d'effet dans les petites populations alors que les grandes populations sont plus différenciées pour les allozymes quand la sélection est hétérogène entre populations. Globalement, nos résultats sont plutôt en accord avec les prédictions théoriques ; certains résultats sont néanmoins inattendus. En particulier, la perte de diversité globale a été très importante (de l'ordre de $20 \%$ en quatre générations), même dans les métapopulations de 2000 individus.

diversité génétique / évolution expérimentale / conservation / petites
populations / précocité

\section{INTRODUCTION}

Genetic resources of crop plants and of their wild relatives have been collected and stored in gene banks for several decades [9]. Some of these banks contain very large numbers of samples and their evaluation, management and utilisation raise an increasing number of questions. From a biological point of view, the main questions concern (i) the tools to evaluate both neutral and potentially non-neutral quantitative genetic diversity, and (ii) regeneration methods allowing maintenance of the initial diversity, prevention of the accumulation of deleterious mutations, and avoidance of the "evolutionary freeze" of populations removed from their natural environment [18]. Similar questions arise regarding the conservation of populations of rare species. The lack of genetic variability associated with small population sizes and, possibly, the accumulation of deleterious mutations are, indeed, thought to be major factors driving small populations to extinction, although these issues are controversial [11].

To address some of these questions a dynamic management of genetic diversity has been suggested $[1,18,33]$. Such a management involves in situ or ex situ maintenance of evolving metapopulations. The metapopulation concept was introduced by Levins [23] as a population of populations connected by migration events and subject to extinction and recolonisation events. The 
aim of such a management is to maintain as much diversity as possible at the metapopulation level, and even to create new genetic combinations while letting the populations evolve and adapt to their local environment [18]. For crop species, artificial populations need to be created, whereas for wild species both in situ and ex situ management could be considered. For instance, a dynamic management of genetic resources was established in 1984 for winter wheat, a selfing species $[6,18,21,22,25,30,31]$.

Genetic diversity roughly comprises two components: neutral diversity, which, by definition, is not under selection in the study environment at a given time, and non-neutral diversity. Potential users of genetic resources are mainly interested in maintaining and evaluating diversity for agronomic traits such as morphology, seed production or pest resistances, which in most environments are under selection. These traits are most likely also related to fitness in wild populations. The measurement of these traits, however, is time-consuming and the trait values can be very dependent on the environment in which they are measured. In contrast, neutral diversity is easier to assess since numerous molecular markers are available and their expression is not dependent on the environment. The theory about the structure of neutral diversity in a metapopulation is furthermore well established (reviewed in [14]) and the neutral diversity of a population can be thought of as a reservoir for potential future adaptation [11]. However, the relationship between the patterns of neutral and selected diversities in a metapopulation depends on numerous factors such as population sizes, effective recombination and type of selection [17]. In this paper, we report on an experiment under controlled conditions that was designed to investigate the effect of different metapopulation managements on the evolution of both allozyme and quantitative genetic variability.

Two experiments were set up to investigate how to maintain or create genetic diversity in artificial and/or managed populations of plants. Our goal was to test the effects of population sizes, selection regime and extinction/recolonisation events in a selfing species. We set up an extinction/recolonisation experiment to mimic three contrasted situations of $i n$ situ management: a habitat with no disturbance, compared with two that experience disturbances; one with high likelihood of recolonisation, and the other with low likelihood of recolonisation. The comparison of the evolution of diversity among the various treatments could help us design and maintain populations for conservation purposes.

\section{MATERIAL AND METHODS}

The study species is the small annual Arabidopsis thaliana (Brassicaceae). It is mainly selfing with natural levels of outcrossing of $1.2-2.2 \%$ [39]. It was chosen for its short life cycle, which in the greenhouse can last as little as two months, and for the large amount of knowledge available on its genetics and physiology [34]. 


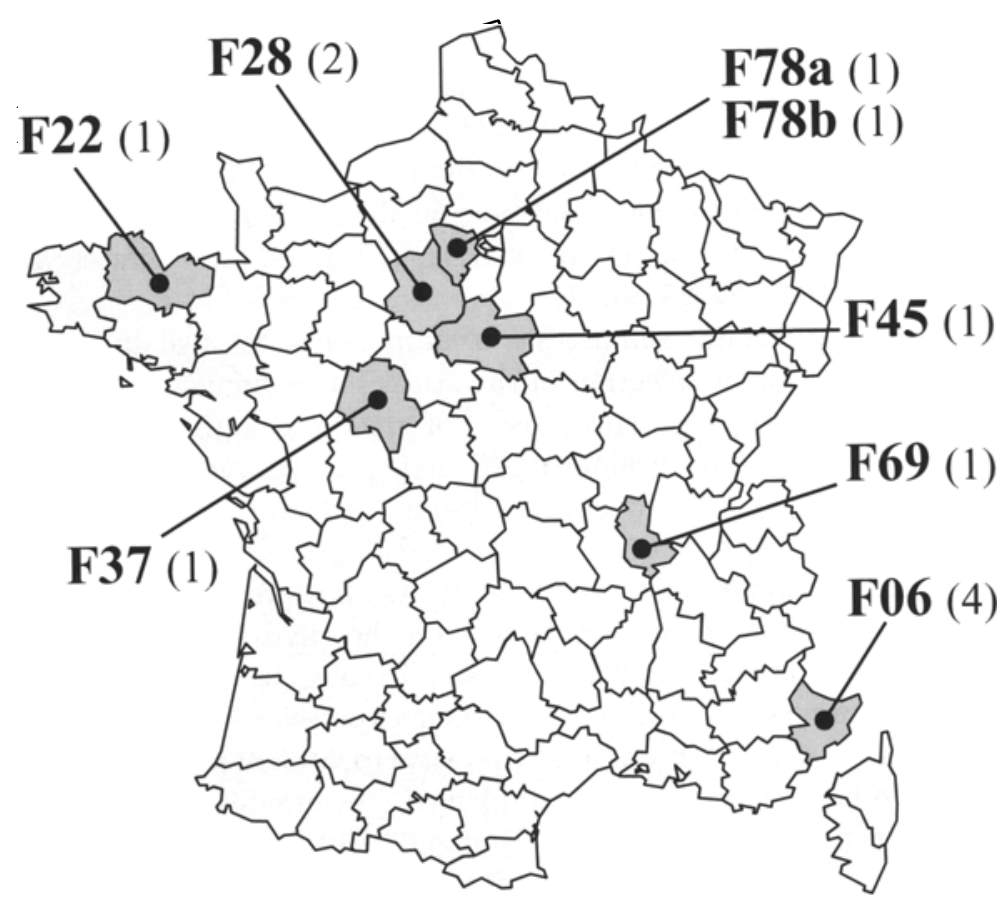

Figure 1. Geographic origin of the parental lines. The names of the parental populations are in bold, followed by the number of parental individuals coming from these populations (in brackets). The GB population (two parental lines) originates from Wales (UK).

\subsection{Initial composition of the metapopulations}

Fourteen lines of $A$. thaliana collected in nine natural populations (Fig. 1) and the nw77 male sterile mutant from the Nottingham Arabidopsis Stock Centre $^{1}$ were used as parental lines of the metapopulations. Male sterility is due to a single point nuclear recessive mutation, which causes the absence of petals, stamens and pollen production. The male sterile mutant was introduced in the populations to maintain some level of outcrossing (see below). The natural parental lines were chosen for their flowering synchrony and for their differences in electrophoretic patterns at five allozyme loci. To create the initial populations, parental lines (Tab. I) were control-crossed by hand following the protocol described on Table II. The result of the crosses was checked by analysing the patterns of the F1 individuals at the five allozyme loci which

${ }^{1}$ Male sterile mutant: mutant NW77 Pistillata, Background Ler. Mutagen EMS, locus pi, allele pi1, Map position 5-23. The Nottingham Arabidopsis stock centre, Department of Life Science, University of Nottingham, University Park, Nottingham NG7 2RD, UK. 
Table I. Allozymic composition of the initial parental lines (for a given locus, each number represents a particular allele). Indices indicate the different individuals within a population.

\begin{tabular}{|c|c|c|c|c|c|}
\hline Parental lines & IDh & LAP-2 & AcPh 1 & AcPh 2 & SkDh \\
\hline F22 & 2 & 4 & 2 & 2 & 1 \\
\hline F28 $1,2_{1,}$ & 2 & 4 & 1 & 1 & 1 \\
\hline GB $_{1}$ & 1 & 4 & 2 & 2 & 1 \\
\hline GB $_{2}$ & 2 & 4 & 3 & 2 & 1 \\
\hline $\mathrm{F}_{1,2,3,4}$ & 2 & 1 & 1 & 2 & 2 \\
\hline F78a & 2 & 4 & 2 & 2 & 1 \\
\hline F78b & 2 & 4 & 2 & 1 & 1 \\
\hline F37 & 2 & 2 & 2 & 1 & 1 \\
\hline F45 & 2 & 3 & 2 & 2 & 1 \\
\hline F69 & 2 & 4 & 2 & 2 & 1 \\
\hline NW77 & 2 & 4 & 2 & 2 & 1 \\
\hline
\end{tabular}

Table II. Crosses at the origin of the F2 and their contribution to the first generation.

\begin{tabular}{|c|c|c|c|}
\hline Cross & Contribution & Cross & Contribution \\
\hline $\mathrm{GB}_{1} \times \mathrm{F} 78 \mathrm{~b}$ & $10 \%$ & $\mathrm{~F} 45 \times \mathrm{F} 28_{2}$ & $10 \%$ \\
\hline$\left(\mathrm{GB}_{1} \times \mathrm{F} 06_{1}\right) \times \mathrm{NW} 77$ & $10 \%$ & $\left(\mathrm{~F} 45 \times \mathrm{F} 06_{3}\right) \times \mathrm{NW} 77$ & $15 \%$ \\
\hline $\mathrm{GB}_{1} \times \mathrm{F} 28_{1}$ & $5 \%$ & $\mathrm{~F} 37 \times \mathrm{F} 06_{4}$ & $5 \%$ \\
\hline $\mathrm{F} 37 \times \mathrm{F} 28_{2}$ & $10 \%$ & $\mathrm{~F} 22 \times \mathrm{F} 28_{1}$ & $10 \%$ \\
\hline $\mathrm{F} 69 \times \mathrm{F} 06_{2}$ & $5 \%$ & $\mathrm{~GB}_{2} \times \mathrm{F} 78 \mathrm{a}$ & $20 \%$ \\
\hline
\end{tabular}

distinguished the parents (Tab. I). F1 individuals were selfed. The resulting F2 seeds were pooled according to the proportions given in Table II and this pool of seeds was used to sow the first generation of each local population of each metapopulation.

\subsection{Experimental designs}

\subsubsection{Treatments}

Two experiments were set up (Tab. III). The first one (hereafter experiment 1, set up at Orsay), aimed to explore the effect of population size and selection regime on the evolution of the diversity in the metapopulations. The second one (hereafter experiment 2, set up at INRA Dijon), was aimed at understanding the effect of different extinction/ recolonisation regimes. In both experiments, migration rate among extant populations was fixed at $2 \%$.

For experiment 1, twelve metapopulations were set up, each containing 20 populations. The two treatments were population size (10, 25 or 100 individuals per population) and selection regime (directional or heterogeneous). Densities were kept independent of the sizes of the populations by growing 
Table III. Number of metapopulations studied for each treatment of each experiment. Each metapopulation was made of 20 local populations.

\begin{tabular}{|c|c|cc|}
\hline Experiment & $\begin{array}{c}\text { Total number of } \\
\text { metapopulations }\end{array}$ & \multicolumn{2}{|c|}{ Selection regime } \\
Directional & Heterogeneous \\
\cline { 1 - 1 } Orsay (No extinction) & 12 & & \\
\cline { 1 - 1 } Population size $(N)$ & & 2 & 2 \\
-100 & & 2 & 2 \\
-25 & & 2 & 2 \\
-10 & 6 & & - \\
Dijon $(N=100)$ & & 2 & - \\
\hline Extinction regime & & 2 & - \\
- No extinction & & 2 & \\
- Migrant pool & & \multicolumn{2}{c}{} \\
- Propagule pool & & & \\
\hline
\end{tabular}

the plants in pots of areas $26.4 \mathrm{~cm}^{2}$ (10 plants), $86.25 \mathrm{~cm}^{2}$ (25 plants) and $350 \mathrm{~cm}^{2}$ (100 plants). Two metapopulations were grown for each population size $\times$ selection regime combination. The selection for a short life cycle was applied by stopping any watering as soon as the first fruits were mature in one of the two replicate metapopulations. In the directional selection regime, each population of each replicate metapopulation was selected for a short life cycle. In the heterogeneous selection regime, each metapopulation was split into two groups of ten populations: one group being selected for a short life cycle, the other being allowed to grow and flower as long as necessary. Populations were assigned to either group at the first generation. Within each metapopulation, all populations were allowed to exchange genes randomly by way of the $2 \%$ migrants.

For experiment 2, metapopulations contained 20 populations of 100 individuals grown in the same large pots as in experiment 1 and all selected for a short life cycle. The treatments consisted of three different extinction-recolonisation regimes: 1) no extinction (as in experiment 1), 2) extinction and recolonisation from a large genetic basis (100 individuals from five different populations drawn at random within the metapopulation), corresponding to a migrantpool pattern [38],3) extinction and recolonisation from a narrow genetic basis (five individuals from one population drawn at random), corresponding to a propagule-pool pattern [38]. The local extinction rate was $25 \%$ for metapopulations with extinctions, i.e. each generation five populations randomly chosen from 20 were eliminated, and replaced by recolonisers. Two replicate metapopulations were grown for each treatment. 


\subsubsection{Experimental conditions of each generation}

In the absence of any female advantage, and as male-sterility is determined by a single recessive gene, the proportion of male-sterile individuals is expected to decrease by half each generation. In order to ensure a minimum outcrossing rate of $10 \%$, for each non-extinct population, and whenever possible, $10 \%$ of the seeds used to grow the following generation were harvested from malesterile individuals of that population. Thus, the average frequency of malesterile individuals among newly produced seeds was expected to be kept at $5 \%$. About $88 \%$ of the seeds for the next generation were harvested from local hermaphrodites. The remaining average $2 \%$ were migrants drawn at random in another single, randomly chosen, population of the metapopulation. In experiment 2, extinct populations were recreated as described above.

Seeds were sown on a regular grid, watered with a solution containing $0.15 \%$ fungicide (Dericlor, Ciba Geigy) and left for one week in the dark at $4{ }^{\circ} \mathrm{C}$ to break dormancy. This cold treatment was stopped in experiment 2 after the third generation. After germination, the plants were grown in a controlled compartment of a greenhouse under a $16 \mathrm{~h} \mathrm{light} / 8 \mathrm{~h}$ dark photoperiod, a temperature varying between $15{ }^{\circ} \mathrm{C}$ night and $20^{\circ} \mathrm{C}$, day and were watered twice a week. In order to avoid local environmental effects, the pots were regularly moved around during the growing period. Two or three times during flowering, flowers of male-sterile individuals were gently rubbed with the maximum number of male-fertile flowers of the population to ensure seed production.

Once watering of the plants was stopped, they were left to dry. Fruits of male-sterile individuals were harvested separately and the rest of the population was harvested as a whole. Seeds were then stored in the dark at $4{ }^{\circ} \mathrm{C}, 10 \%$ humidity.

\subsection{Measurements}

\subsubsection{Allozymic diversity}

In the fourteen parental lines we observed four alleles for the Leucine Amino Peptidase locus (LAP-2), three alleles for the Acid Phosphatase 1 locus (AcPh 1) and two alleles for the Acid Phosphatase 2 locus ( $\mathrm{AcPh} 2$ ), the Shikimic Dehydrogenase locus (SkDh) and the Isocitric Dehydrogenase locus (IDh). Enzyme polymorphism was assessed on IDh, LAP-2, AcPh 1 and $\mathrm{SkDh}$ at the fourth generation for experiment 1 , and on the LAP-2, AcPh 1 and 2 loci every generation for experiment 2 .

In experiment 1 , we sampled ten individuals from five populations in each metapopulation with 25 or 100 individuals per population, and seven individuals from six populations in each metapopulation with 10 individuals per population. In experiment 2, 10 individuals per population were sampled except for populations under narrow-basis recolonisation for which the total population of five individuals was analysed. The origin and number of populations analysed 
varied from generation to generation (38 populations in Generation 1 (G1), 44 in $\mathrm{G} 2,40$ in $\mathrm{G} 3,56$ in G4).

Enzyme extracts were obtained from leaves of four week-old plants. For experiment 1 (performed in Orsay), extracts were migrated on a $13 \%$ starch gel in a Lithium borate buffer (adapted from [40]) to reveal the polymorphism for the LAP and AcPh enzymes, and on a $12.5 \%$ starch gel in a Tris citrate buffer (adapted from [32]) for the SkDh and IDh enzymes. For experiment 2 (performed in Dijon), foliar isoenzymes were separated by electrophoresis on a polyacrylamide gel using the method described by Ornstein [29] and Gasquez and Compoint [13] in a discontinuous system with pulse power. Detailed specifications of laboratory techniques are given in [3].

\subsubsection{Genetic analyses}

Nei's unbiased estimator of gene diversity [28] was calculated for populations and metapopulations using the Fstat software [15].

Within-population diversities per locus were estimated as:

$$
H_{s}=\frac{\tilde{n}}{\tilde{n}-1}\left(1-\sum \overline{p_{i}^{2}}-\frac{H_{o}}{2 \tilde{n}}\right)
$$

where $\tilde{n}$ is the harmonic mean of the numbers of individuals per population, $\overline{p_{i}^{2}}$ is the average over populations of the squared frequency of each allele at a given locus, and $H_{o}$ is the average observed heterozygosity at this locus. The average diversity is calculated as the average $H_{s}$ over loci.

Overall gene diversities per locus are estimated as:

$$
H_{t}=1-\sum\left(\bar{p}_{i}\right)^{2}+\frac{H_{s}}{\tilde{n} n_{p}}-\frac{H_{o}}{2 \tilde{n} n_{p}},
$$

where $n_{p}$ is the number of populations, $\bar{p}_{i}$ the average frequency of each allele at a given locus over populations, and $H_{o}$ is the observed heterozygosity at this locus. The average diversity is calculated as the average $H_{t}$ over loci.

Weir and Cockerham's estimators of $F_{i s}$ and $F_{s t}$ [45] (hereafter named $\hat{F}_{i s}$ and $\hat{F}_{s t}$ ) were also calculated with the Fstat software. The test for significant difference of these $F$-values from zero was performed by permuting individuals over populations. No standard errors were calculated due to the small number of loci studied.

Given the initial composition of the metapopulations, the expected diversities were $H_{t 0}=H_{s 0}=0.35$ for experiment 1 and $H_{t 0}=H_{s 0}=0.47$ for experiment 2 at the first generation. The difference between experiments is due to the difference in the set of loci studied. Expected $F_{s t}$ values were zero since populations were created from the same seed pool, and the expected $F_{i s}$ value was 0.40 in experiment 1 and 0.42 in experiment 2 . 


\subsubsection{Precocity}

As conditions in the greenhouse may vary throughout the year, a direct comparison of flowering phenology in number of days between germination and flowering could be misleading. To minimise the environmental effect, populations from several generations were compared simultaneously. Thus, in experiment 2 , the efficiency of the selection for precocity was assessed for the first three generations on a sub-sample of three populations per metapopulation leading to six metapopulations $\mathrm{X}$ three populations $\mathrm{X}$ three generations +10 samples for generation $1=64$ samples. From each sample, 50 seeds were sown in plastic pots $(17.5 \times 13 \times 5.5 \mathrm{~cm})$. Every day for 90 days, the proportions of plants in the following stages were noted: cotyledons, rosette, bolting, flowering, green pods and mature fruits.

The cumulative evolution of flowering frequency over time in days (D) was then fitted to the following logistic model:

$$
\text { cumevolf }=\frac{K}{[1+(K-1) \exp (-r(D-i))]},
$$

where $i$ is the estimated parameter for the time lag before first flowering, $r$, the synchrony of flowering and $K$, the proportion of flowering when the experiment was stopped. The goodness of fit was assessed using a $R^{2}$. Applying this model to each of the 64 samples, all $R^{2}$ values ranged between 0.873 and 0.997 with a mean value of $0.972 \pm 0.023$ and were all highly significant.

The generation effect was then tested using one-way ANOVA applied individually to each of the three estimated parameters described above.

\subsection{Theoretical predictions}

\subsubsection{Effect of selection regime on local neutral diversity (Experiment 1)}

Selection increases the variance in offspring number [16], thus reducing effective population size. The reduction in effective population size should only depend on the strength of selection, not on it being homogeneous or heterogeneous. Thus, for those loci unlinked to selected ones, we do not expect any effect of the selection regime on local diversity. Alternatively, one could consider that when all populations are selected for precocity, selection is stronger and thus effective population sizes are smaller in the homogeneous selection treatment compared with the heterogeneous treatment. In this case, we expect less genetic diversity in the homogeneous selection regime. Overall, and for a given population size, we expect local genetic diversity in the homogeneous selection regime to be either the same or smaller than in the heterogeneous selection regime. 


\subsubsection{Effect of selection regime on differentiation among populations for neutral markers (Experiment 1)}

For a given population size, effective migration rate should be lower in the heterogeneous selection regime, as on average immigrants are not locally adapted [7]. Although differentiation among populations for unlinked neutral loci is not expected to be highly influenced by the type of selection, if it happens to be it should be such that populations are more differentiated under the heterogeneous selection regime.

Hitch-hiking might also affect the evolution at neutral loci in linkage disequilibrium with selected ones. Directional selection tends to homogenise populations for those loci undergoing selection and linked neutral loci, whereas heterogeneous selection promotes differentiation. For those loci, we thus also expect to find more differentiation under the heterogeneous selection regime.

Overall, for a given population size, we thus expect more differentiation under the heterogeneous selection regime.

\subsubsection{Effect of selection regime on global neutral diversity (Experiment 1)}

Assuming that the strength of selection is the same in the two selection regimes, the sole effect of selection is on differentiation among populations, expected to be stronger under heterogeneous selection. We thus expect global diversity to be larger under this regime. The pattern is reinforced if we assume that the strength of selection is greater under homogeneous selection since local diversity would decrease similarly in all populations.

We thus expect global diversity to be smaller in the homogeneous selection regime. This is particularly expected for those genes linked to those determining precocity.

\subsubsection{Effect of population size on neutral diversity (Experiment 1)}

As local population sizes increase, genetic drift is weaker locally. Therefore, at the local level, we expect genetic diversity to increase with local population size. Assuming an equilibrium between migration and drift, the shape of $F_{s t}$ as a function of $N^{*} m$ indicates that above the value $N^{*} m=1$, the level of differentiation among populations is expected to be low [5]. For a complete selfer, this "threshold" value is $N^{*} m=3$. In experiment 1 , the rate of seed immigration is $2 \%$, thus the numbers $N m$ of migrants per generation are 0.2 , 0.5 and 2 for the three population sizes. This is probably less than the threshold value above which migration is expected to counteract drift in our experimental system, as selfing rate was in fact large, albeit male-sterility. Thus we expect large differentiation among populations to build up, all the more so for low effective sizes. 
In finite populations, selection has more impact on the frequency of a favoured allele than drift only if $N_{e}{ }^{*} s>1$ where $s$ is the selection coefficient [16]. We therefore expect response to selection to be stronger for larger population sizes, at least in the directional selection regime. The increase in diversity as populations become larger should thus be smaller than expected with the purely neutral model. In the heterogeneous selection regime, the effective number of migrants should also increase less than with the purely neutral model, since, as already stated above, migrants on average are not locally adapted. Overall, the reduction in effective size for neutral genes due to selection at other loci is expected to increase with population size, at least in the homogeneous selection regime, reducing the direct effect of diminishing drift with increased population size. We thus expect a slight decrease in differentiation with increased population size.

\subsubsection{Effect of local extinctions and recolonisation treatment on neutral diversity (Experiment 2)}

Local extinctions have in theory two effects [43,46]. First, they decrease the total metapopulation size and thus the overall genetic diversity should be lower compared to the no extinction treatment. Second, they create a bottleneck at recolonisation, and thus increase the amount of differentiation among populations. At the same time, they increase gene flow among populations, thus acting against differentiation. In the first extinction treatment, recolonisations occur by sampling 100 migrants from several populations, so that the main effect of extinction is to increase gene flow. In the second extinction treatment, only five individuals, sampled from a single population, are allowed to act as recolonisers. Thus the main effect of this treatment is a bottleneck. We thus predict that the stronger differentiation at equilibrium should be observed in the second ("narrow-basis") extinction treatment, followed by the no extinction treatment, followed by the large-basis extinction treatment. Total diversity should be larger in the no extinction treatment.

\subsubsection{Effect of local extinctions and recolonisation treatment on response to selection (Experiment 2)}

Response to selection is expected to increase with total effective metapopulation size. It should thus be larger in the no extinction treatment, followed by the extinction treatment with a large genetic basis recolonisation, followed by the bottleneck treatment. Note however that enhanced gene flow, as experienced in the extinction regime with large base recolonisation, might at the same time locally increase the evolutionary potential, and thus favour local adaptation [12]. Thus, no clear prediction can be made without further modelling. 


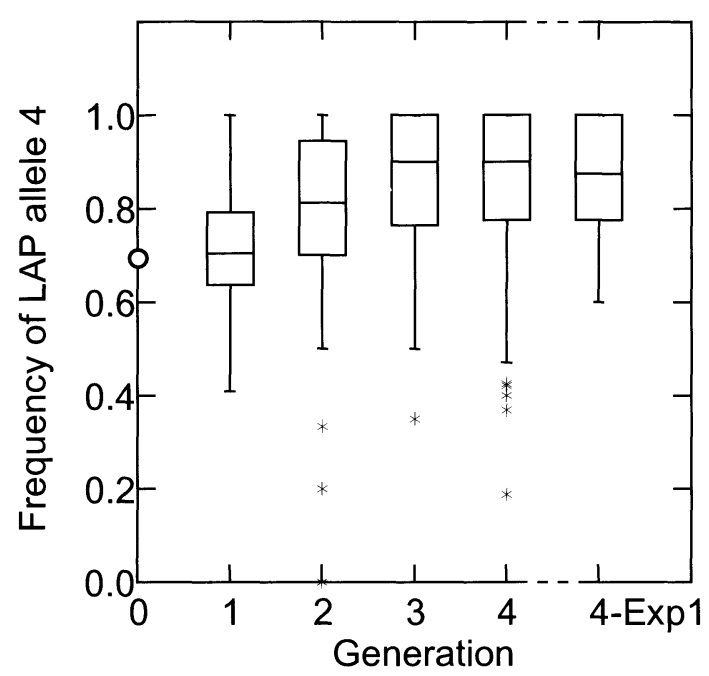

Figure 2. Box plot graph representing the evolution of the frequency of allele 4 of the LAP-2 during the first four generations in experiment 2 and at the fourth generation in the large metapopulations of experiment 1 . The circle on the Y-axis indicates the initial frequency of the allele. The boxes represent the interquartile range, and asterisks represent outliers. Data are represented on a per population basis, independently of the metapopulation.

\section{RESULTS}

We present results for the first four generations of the experiments.

\subsection{Allozyme variability}

\subsubsection{Allelic and genotypic frequencies}

Considering the two experiments, allele 4 of the LAP-2 increased in frequency between the first and the fourth generation in 16 out of the 18 metapopulations. This is significantly different from the $50 \%$ increase, $50 \%$ decrease expected with drift (rank test $P<0.001$ ). Its increase in frequency is significant in all metapopulations (Kruskal-Wallis statistics $=31.21, P<0.0001$ for a generation effect) in experiment 2 where it was estimated at each generation. The frequency of this allele was not significantly different between the two experiments at the fourth generation when only metapopulations with 100 individuals per population were considered $(P=0.72)$ (Fig. 2). There was no significant effect of the treatment on the frequency of this allele in either experiment $(P=0.56$ in experiment 1 and $P=0.95$ in experiment 2$)$. This allele was also the most frequent in natural populations of Arabidopsis thaliana analysed 
in our laboratory (data not shown), and was present in seven out of nine populations initially used as a basis for the metapopulations (Tab. I). Significant changes in allele frequencies were observed for the other enzymatic systems, but there was no obvious relationship between the level of significance of the tests, the direction of change and the population size or selection treatment (data not shown).

Removing the LAP-2 locus from the following analyses increased slightly the values of $\hat{F}_{s t}$, but it did not modify the general pattern (data not shown). The results over all loci will therefore be discussed.

\subsubsection{Within-population diversity}

Within-population diversity decreased in all metapopulations. After four generations, the average decrease was about $66 \%$ in experiment 1 (Fig. 3) and $55 \%$ in experiment 2 (Fig. 3, G4), assuming the expected initial diversities.

In experiment 1 , an ANOVA performed on $H_{s}$, the local diversity averaged per locus and per population for each metapopulation suggests no effect of either population size $\times$ selection regime $(p=0.15$, one-way ANOVA). However, a Duncan's Multiple Range Test performed on average $H_{s}$ per metapopulation suggests that the lowest diversity was retained in the treatment with homogeneous selection and smallest population size $\left(H_{s}=0.16\right.$ for this treatment, and $H_{s}=0.23$ to 0.25 for the other treatments).

In experiment 2, while the effect of generation on local diversity was highly significant $(P=0.0001)$, there was no significant effect of the extinction treatment $(P=0.75)$ (two-way ANOVA with generation and treatment as independent variables). The decrease occurred during the first three generations, as average local diversities of generations three and four were not significantly different, whereas $H_{s}$ of generation one was significantly larger than diversity of generation two, itself significantly larger than diversity of generations three and four (Duncan's Multiple Range Test of procedure ANOVA, SAS).

\subsubsection{Population structure: $\hat{F}_{s t}$ and $\hat{F}_{i s}$}

Population structure increased over time and was highly dependent on the treatment (Fig. 4). Two-way ANOVA with generation and treatment effects as independent variables in experiment 2 showed that $\hat{F}_{s t}$ was significantly influenced by both the generation and the extinction treatment $(P=0.003$ for generation and $P=0.016$ for treatment). In this experiment, the increase was greatest in the no extinction treatment (average $\hat{F}_{s t}=0.30$ ), and smallest in metapopulations with extinction and large base recolonisation (average $\hat{F}_{s t}=$ $0.13)$.

In experiment 1 , the amount of differentiation was explained by local population sizes $\left(P<0.0001\right.$, two-way ANOVA on average $\hat{F}_{\text {st }}$ per metapopulation, with population size and selection regime as independent variables). Thus, 


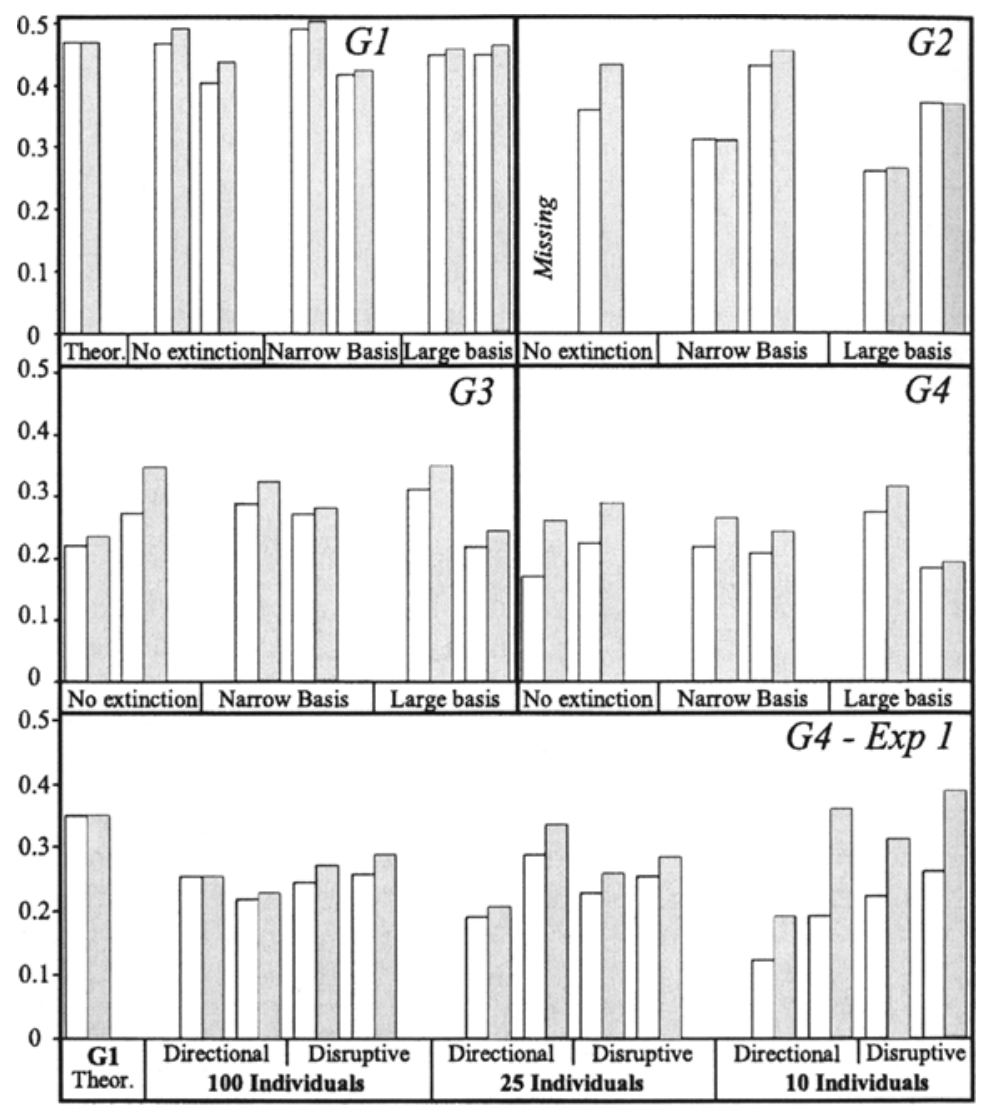

Figure 3. Estimates of gene diversity during the first four generations of experiment 2 (G1 to G4) and at the fourth generation in experiment 1. Data are missing for the second generation in the no extinction treatment. $\square$ Within-population diversity $H_{s}$; Total diversity $H_{t}$; Theor.: theoretically expected at the first generation.

metapopulations with large population sizes were slightly differentiated (average metapopulation $\hat{F}_{s t}$ value of 0.03 ), whereas metapopulations with small population sizes were highly differentiated (average metapopulation $\hat{F}_{s t}$ value of $0.45)$. There was no significant effect with treatment as main effect $(P=0.99)$ but the interaction between treatment and local population size was significant $(P=0.03)$. For population sizes of $100 \hat{F}_{s t}$ was larger under the heterogeneous selection regime (average metapopulation $\hat{F}_{s t}=0.12$ and $\hat{F}_{s t}=0.02$ in the heterogeneous and in the homogeneous selection regimes, respectively; $P=0.04$, one-way ANOVA on $\hat{F}_{s t}$ per metapopulation with population size of 100 , with selection regime as the independent variable).

All $\hat{F}_{i s}$ values were large and highly significant. The mean $\hat{F}_{i s}$ was $0.788 \pm$ 0.03 across metapopulations. There was a tendency for $\hat{F}_{i s}$ to increase over 


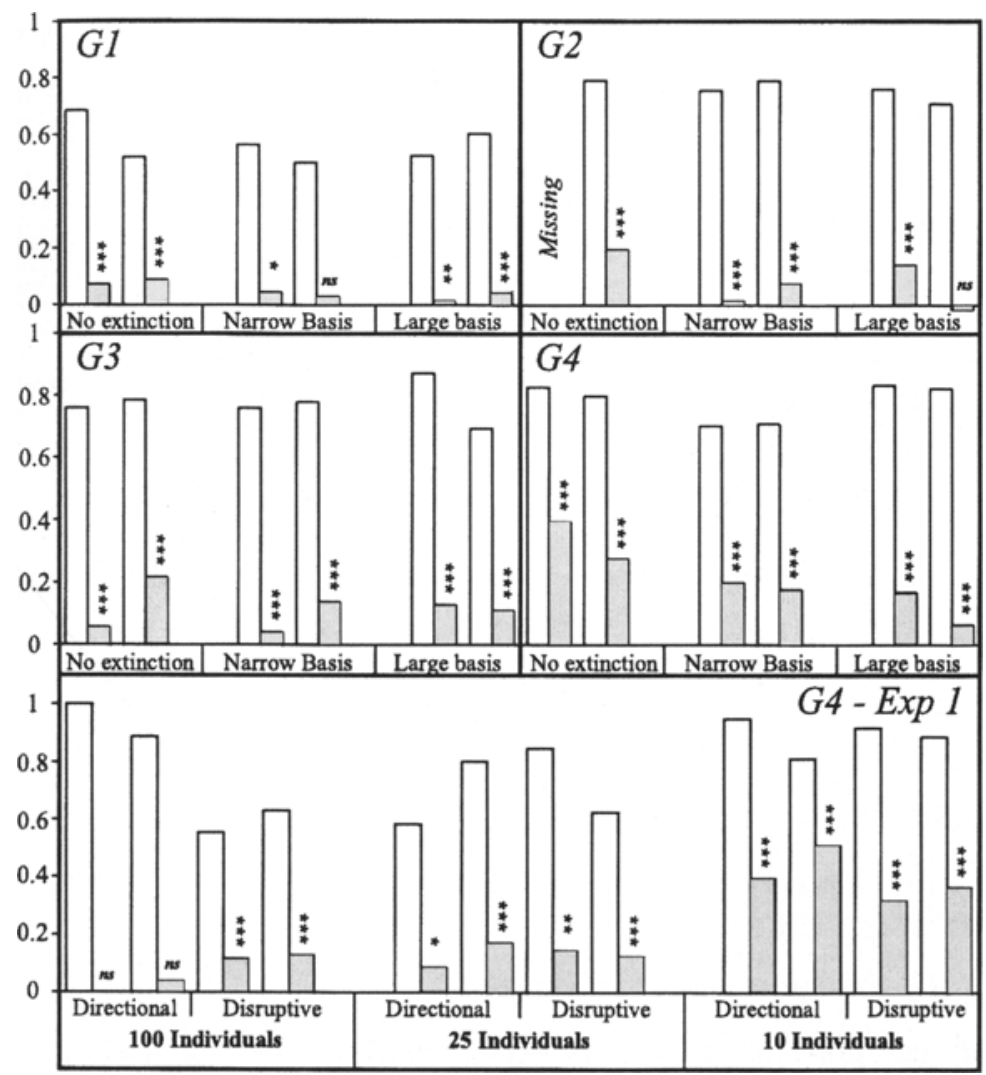

Figure 4. Estimates of multilocus $\hat{F}_{s t}(\mathbf{D})$ and $\hat{F}_{i s}(\square)$ during the first four generations of experiment 2 (G1 to G4) and at the fourth generation in experiment 1 . $\hat{F}_{i s}$ values are all highly significant. Levels of statistical significance for $\hat{F}_{s t}$ are noted as $\boldsymbol{n s}$ : $P>0.05, *: P<0.05,{ }^{* *}: P<0.01,{ }^{* * *}: P<0.001$.

generations as shown for generations one to four in Figure 4 for experiment 2. Two-way ANOVA of average (local) $\hat{F}_{\text {is }}$ per metapopulation shows no effect of treatment in either experiment $(P=0.28$ for selection regime and $P=0.26$ for local population size in experiment 1 and $P=0.146$ for experiment 2). It confirms the generation effect on $\hat{F}_{i s}$ for experiment $2(P<0.0001)$, but no evolution of $\hat{F}_{i s}$ is observed after generation two in this experiment $(P=0.24)$. Assuming that $\hat{F}_{i s}$ values are therefore close to equilibrium at the fourth generation we estimated the outcrossing rates in both experiments [16]. Estimated outcrossing rates are consistent between experiments $(0.12 \pm 0.03$ in experiment 1 and $0.12 \pm 0.02$ in experiment 2). 

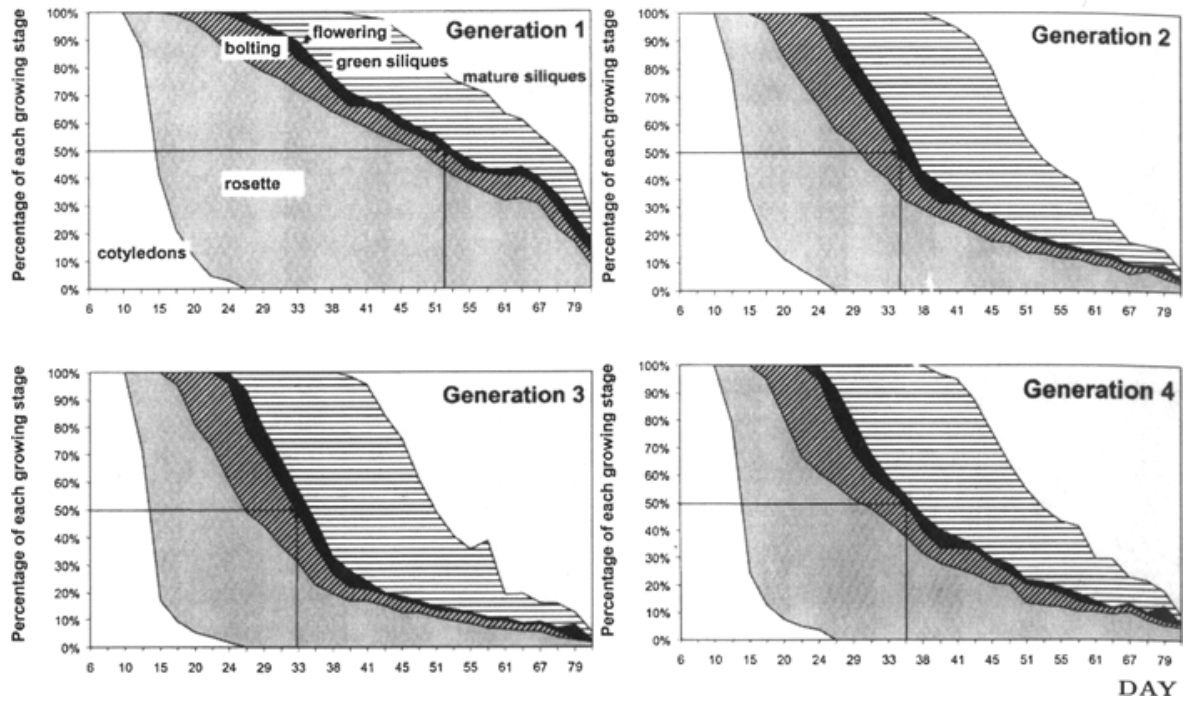

Figure 5. Evolution over time of the percentage of six growing stages (cotyledons, rosette, bolting, flowering, green pods and mature fruits) at the first generation (500 plants) and at the three subsequent generations (900 plants each, pooled over treatments).

\subsubsection{Global population diversity}

Global metapopulation diversity decreased in all metapopulations (Fig. 3). After four generations, the average decrease was $20 \%$ in experiment 1 and $17 \%$ in experiment 2 . Consistently, in experiment 2 the generation effect was significant $(P=0.0007)$ in a two-way ANOVA with extinction regime and generation as independent factors. The extinction treatment contrarily was not significant $(P=0.60)$. There was no effect of the treatment on metapopulation diversity in experiment 1 either $(P=0.30$ and 0.46 for selection regime and local population size treatments in two-way ANOVA).

\subsection{Precocity}

The results of the selection experiment 2 are given in Figure 5. Selection was efficient since $50 \%$ of the plants were still at the rosette stage 52 days after sowing for plants of the first generation, whereas this period was reduced to 36 days after three generations of selection. The overall shape of the graphs however suggests that the response to selection mostly occurred after the first generation of selection. Most reduction in the life cycle was due to a shortening of the rosette stage. Two-way ANOVA with generation and extinction treatment as independent variables shows a strong generation effect on synchrony (parameter $r, P<0.001$ ) as well as a significant increase in the final 
proportion of flowering plants (parameter $K, P=0.036$ ), while there was no significant change in time to appearance of the first flowering plant (parameter $i, p=0.29)$. The only significant effect of treatment on response to selection was that extinction with large base recolonisation retained significantly more non-flowering plants than the two other treatments.

\section{DISCUSSION}

The two experiments were designed to investigate changes in levels and distribution of genetic diversity under contrasted metapopulation regimes. This knowledge may prove useful for the management of metapopulations for the conservation of genetic variation. The maintenance of genetic diversity at the population and the metapopulation levels depends on effective population and metapopulation sizes. The effective size of a population is usually lower than its census size. Frankham [10] provides a very general figure of 0.11 for wild populations. The correlation between the effective size of a population and its census size depends on factors such as selfing rate, selection regime and migration. The effects of the factors acting in our experiments are detailed in the material and methods section. In general, we expected more genetic diversity to be maintained for large effective sizes.

\subsection{Evolution of allelic frequencies}

Our estimation of neutral genetic diversity is based on polymorphism at four allozymic loci. It is generally considered that allozymes behave as neutral markers. This issue, however, is somewhat controversial: a positive correlation between heterozygosity for allozymic loci and fitness-related traits such as viability or growth rates is reported for organisms as different as wild oat [4], a number of tree species (e.g. [8,41]), a fish [27] and shellfish [19,42]. Similarly, the decline in the proportion of heterozygotes with inbreeding is usually lower than expected (e.g. Rumball et al. [36] in Drosophila).

In our experiment, we observed an increase in the frequency of allele 4 of LAP-2 in most metapopulations. This might suggest that this allele is under selection in our experiment. LAP is an enzyme active in the cytosol which participates in the turnover of proteins [2]. Koehn et al. [19] observed a correlation between allele frequencies at the LAP-2 locus and salinity and/or temperature in oyster and suggested that one allele might be under selection at this locus. However, the correlation was no longer significant in the same organism in a later study [37]. Another explanation would be that allele 4 of LAP-2 is in linkage disequilibrium with determination of precocity. It is easy to see how subsampling at the foundation of the lines could have generated such linkage disequilibrium. No correlation was detected between the frequency of allele 4 and precocity in the ten F2 lines used to found all metapopulations (data 
not shown). However, because of the high frequency of that allele in founder lines, our ability to detect linkage disequilibrium with genes for precocity is diminished. Allozyme loci have been convincingly shown to be under direct and directional selection in a few cases (e.g., Watt [44] for selection favouring particular alleles of phospho-gluco isomerases in butterflies). Further work is needed in our experimental system before we can suggest the most likely explanation for the pattern observed with LAP-2.

Below we summarise the results and compare them with our original predictions.

\subsection{Neutral diversity}

\subsubsection{Within-population diversity}

After four generations of management, we observed an overall decrease in local diversity for the allozymic markers.

\subsubsection{Effect of local population sizes (Experiment 1)}

Prediction: The local genetic diversity should decrease with the sizes of local populations under no selection.

Test: Overall, there was no significant effect of the population size. It could be that, because selection is more efficient at larger population sizes, and thus because the reduction of effective size by selection is greater for such sizes, this effect partly compensates the effect of decreasing population size so that overall there is little effect of population sizes. The only visible effect is that the smallest local genetic diversity was observed for one of the selection regimes with the smallest population size, and this size $\times$ selection regime treatment was found to be different from the others using the Duncan multiple range test. Moreover, the largest range of variability was observed among metapopulations of small populations, indicating that the level of local diversity was prone to larger variations from generation to generation when local populations were smaller.

\subsubsection{Effect of selection regime (Experiment 1)}

Prediction: the local genetic diversity in the homogeneous selection regime should be less than or equal to that in the heterogeneous selection regime.

Test: Overall, there was no significant effect of the selection regime on local diversity. However, the smallest local genetic diversity was observed for one of the three metapopulation types with the homogeneous selection regime, and this treatment was found to be different from the others using the Duncan multiple range test (same as above). 


\subsubsection{Effect of local extinctions (Experiment 2)}

Prediction: local diversities should be larger in the no extinction treatment

Test: Unexpectedly, there was no significant effect of local extinctions on local diversity. In particular, populations of metapopulations undergoing local extinctions and recolonisation with a narrow-basis were no less variable than populations experiencing a stable environment, an unexpected result. We will discuss this result when considering global diversity.

\subsubsection{Population differentiation}

\subsubsection{Effect of local population sizes (Experiment 1)}

Prediction: Large differentiation among populations should build up, all the more so for low effective sizes.

Test: We indeed observed a highly significant differentiation among populations at the fourth generation. The influence of population size on population structure was very strong and in the expected direction, suggesting that selection did not significantly decrease effective size at neutral loci in large populations.

\subsubsection{Effect of selection regime (Experiment 1)}

Prediction: For a given population size, differentiation under the heterogeneous selection regime should be stronger compared to the homogeneous selection.

Test: There was little effect of selection regime, except for metapopulations with large population sizes, for which differentiation under the heterogeneous selection regime was indeed stronger compared to the homogeneous selection regime. Most likely selection was more efficient at large population sizes, so that the effect of the selection regime was more obvious for such population sizes. The larger differentiation in these metapopulations with heterogeneous selection can be explained both by linkage disequilibria and by a weaker adaptation of the migrants, although we have no experimental evidence for this latter hypothesis. Decay of linkage disequilibrium would be retarded by high selfing in our populations.

\subsubsection{Effect of local extinctions (Experiment 2)}

Prediction: The stronger differentiation at equilibrium should be observed in the narrow-basis extinction treatment, followed by the no extinction treatment, followed by the large-basis extinction treatment.

Test: The smallest differentiation was indeed observed in metapopulations with a large basis for recolonisation, a result consistent with predictions. However, and unexpectedly, significantly less differentiation among populations was 
observed in the narrow-basis recolonisation treatment compared to the no extinctions. This could be explained by the fact that high rates of extinction and recolonisation increase migration rates and more than compensate for the founder effects at recolonisation.

\subsubsection{Global diversity}

\subsubsection{Effect of local population sizes (Experiment 1)}

Prediction: Because the metapopulation size is proportional to local size, the most obvious prediction is that global diversity should increase with local population size.

Test: The level of global diversity was independent of the local population size. As the migration rate is constant, a decrease in local population size both decreases local effective size and increases differentiation among populations. Overall, this could explain why global diversity is not affected by local population size. Moreover, we showed that while differentiation was indeed larger for smaller population size, local diversity was independent on local size (possibly because of selection being more efficient in large populations). So it may not be surprising that global diversity is independent of population size.

\subsubsection{Effect of selection regime (Experiment 1)}

Prediction: Global diversity should be smaller in the homogeneous selection regime, as the local genetic diversity is expected to be less than or equal to that in the heterogeneous selection regime, and so is the amount of differentiation among populations.

Test: This effect of the selection regime is not observed, as the level of diversity is not larger under heterogeneous selection. This is true even in the large metapopulations where differentiation was larger under the heterogeneous selection. More generations might be needed for this effect to build up.

\subsubsection{Effect of local extinctions (Experiment 2)}

Prediction: Total diversity should be larger in the no extinction treatment.

Test: Contrary to our predictions, the global diversity was not affected by extinctions. It could be that because all diversity was present within each population at the beginning of the experiment, the loss of some populations had no immediate effect on diversity. This would also explain why local diversity is not influenced by local extinctions. 


\subsection{Evolution of precocity (effect of local extinctions, Experiment 2)}

Our results on precocity suggest that we effectively selected for more precocious genotypes. The existence of genetic variation for precocity among populations of $A$. thaliana has been reported in many studies (e.g. [20]) and major genes $[24,35]$ and QTLs [26] responsible for this variation are known. In a comparison among 13 populations of $A$. thaliana, Zhang and Lechowicz [47] observed that rosette diameter and its growth rate contributed most to explaining the total variation in flowering time. Here we observed a similar correlated response, since selecting for short life cycles (i.e. early flowering) mainly decreased the length of the rosette stage (Fig. 5).

The significant effect of generation on parameter $r$ of the logistic curve, showing that individuals were more and more synchronous in their flowering behaviour, also illustrates that the efficiency of selection could be linked to a decrease in genetic diversity for this trait.

In general it was difficult to assess what impact selection for precocity had on the level of neutral diversity in our metapopulations. Although most variation for precocity disappeared in the second generation, there was no tendency for neutral diversity to decrease markedly at the same time in experiment 2 .

Response to selection was not influenced by the extinction-recolonisation treatment, suggesting that enhanced gene flow due to local extinctions might bring new variation locally, and compensate for the overall decreased metapopulation effective size that would slow down response to selection. Alternatively, as most of selection response occurred during the first generation, it could be that no variation was left after this generation, so that there would be no further effect of decreased effective size.

\section{CONCLUSION}

Results are only available for the four first generations and it is too early to draw general conclusions useful for conservation of genetic diversity in crop or ex situ conserved species. However, despite the small number of studied loci, several trends emerge from these preliminary results. First, we observed a general decrease (of the order of $20 \%$ ) of neutral genetic diversity in the metapopulations (Fig. 3). This shows that the population sizes we chose were not large enough to maintain efficiently the initial level of diversity, especially regarding the fact that artificial selection was applied. In practise, conservationists often have to tolerate a small decrease of diversity, because maintaining large enough populations is too costly in terms of space and money. Surprisingly, the loss of variability did not depend on population size in our experiment (Fig. 3) after four generations. Thus, a pool of 2000 individuals $(100 \times 20$ for the largest metapopulation) did not seem sufficient to maintain neutral diversity 
efficiently. It could be argued that the artificial selection that was applied on precocity acted to decrease the effective size, so that if one could prevent natural or artificial selection from acting, 2000 individuals might be sufficient to preserve neutral diversity. As the objective of such management programmes is to let populations coevolve with their environment, it is likely (and hoped) that natural selection would occur in any experimental design.

In our experiment, a minimum of 100 individuals per population was necessary to observe an effect of the type of selective pressure on metapopulation structure. Moreover, there was no effect of selection regime on local diversity. This result confirms that, even with large selective pressures, drift overrides selection in small populations. A goal of a dynamic management is to maintain diversity at selected traits, for example by growing the plants in different environments. The large metapopulations in experiment 2 appeared large enough to respond to selection but larger metapopulations might prove necessary if weaker selective pressures are acting.

Finally, population size and selection act together to determine the maintenance of neutral diversity. Both selection and drift decrease the withinpopulation neutral diversity, selection being more efficient in large populations while drift is more efficient in small populations. Maintaining local natural selection in a dynamic management will thus accelerate the loss of neutral genetic diversity, compared to populations undergoing no selection. Heterogeneous selection is expected to counteract this loss of diversity, through its effect on increased differentiation among populations for locally adapted traits. In a selfing species, linkage disequilibria exist at the whole genome level. Thus, the increase in global adaptive diversity through increased differentiation because of heterogeneous selection is also expected to occur for neutral diversity. Assuming populations are connected, local evolutionary potential is restored through rare migration events. In an outbreeding species, selection in different environments will allow the maintenance of adaptive variability, but neutral diversity will be less easily maintained, especially if selection is strong. Thus, a milder selection should be applied in such species.

\section{ACKNOWLEDGEMENTS}

This work was financed by the Bureau des ressources génétiques as well as the Région Bourgogne. We would like to thank L. Saunois, G. Félix, A. Matejicek and M. Schoutith for technical assistance. We are also grateful to P.H. Gouyon and I. Till-Bottraud for initial ideas on these experiments and P. England for input on the final manuscript. This is publication $\mathrm{N}^{\circ}$ ISEM2001-057 of the Institut des sciences de l'évolution. 


\section{REFERENCES}

[1] Allard R.W., The genetics of host-pathogen coevolution: implications for genetic resource conservation, J. Hered. 79 (1990) 225-238.

[2] Bartling D., Weiler E., Leucine aminopeptidase from Arabidopsis thaliana. Molecular evidence for a phylogenetically conserved enzyme of protein turnover in higher plants, Eur. J. Biochem. 205 (1992) 425-31.

[3] Chauvel B., Gasquez J., Relationships between genetic polymorphism and herbicide resistance within Alopecurus myosuroides Huds, Heredity 72 (1994) 336-344.

[4] Clegg M.T., Allard R.W., Viability versus fecundity selection in the slender wild oat, Arvena barbata L., Science 181 (1973) 667-668.

[5] Crow J.F., Kimura M., An introduction to population genetics theory, Harper \& Row, New-York, 1970.

[6] David J.L., Pham J.L., Rapid changes in pollen production in experimental outcrossing populations of wheat, J. Evol. Biol. 6 (1993) 659-676.

[7] Enjalbert J., Goldringer I., David J., Brabant P., The relevance of outcrossing for the dynamic management of genetic resources in predominantly selfing Triticum aestivum L. (bread wheat), Genet. Select. Evol. 30 (1998) S197-S211.

[8] Farris M.A., Mitton J.B., Population density, outcrossing rate and heterozygote superiority in Ponderosa pine, Evolution 38 (1984) 1151-1154.

[9] Frankel O.H., Brown A.H.D., Burdon J.J., The conservation of plant biodiversity, Cambridge University Press, Cambridge, 1995.

[10] Frankham R.F., Effective population size/adult population size ratios in wildlife: a review, Genet. Res. 66 (1995) 95-107.

[11] Frankham R.F., Quantitative genetics in conservation biology, Genet. Res. 74 (1999) 237-244.

[12] Gandon S., Capowiez Y., Dubois Y., Michalakis Y., Olivieri I., Local adaptation and gene-for-gene coevolution in a metapopulation model, Proc. R. Soc. Lond. (Biol.) 263 (1996) 1003-1009.

[13] Gasquez J., Compoint J., Isoenzymatic variations in populations of Chenopodium album L. resistant and susceptible to triazines, Agro Ecosystems 7 (1981) 1-10.

[14] Godelle B., Austerlitz F., Brachet S., Colas B., Cuguen J., Gandon S., Gonyon P.-H., Lefranc M., Olivieri I., Reboud X., Vitalis R., The genetic system, selected genes and neutral polymorphism: Implications for conservation biology, Genet. Select. Evol. 30 (1998) S15-S28.

[15] Goudet J., Fstat version 1.2: a computer program to calculate Fstatistics, J. Hered. 86 (1995) 485-486.

[16] Hartl D.L., Clark A.G., Principles of population genetics, Sinauer Associates, Inc., 1989.

[17] Hastings A., Harrison S., Metapopulation dynamics and genetics, Ann. Rev. Ecol. Syst. 25 (1994) 167-188.

[18] Henry J.P., Pontis C., David J.L., Gouyon P.H., An experiment on dynamic conservation of genetic resources with metapopulations, in: Seitz, A. and Loeschcke, V. (eds.), Species Conservation: A Populational-Biological Approach, Birkhaüser Verlag, Basel, 1991, pp. 185-198.

[19] Koehn R.K., Milkman R., Mitton J.B., Population genetics of marine Pelecypods. IV. Selection, migration and genetic differentiation in the blue mussel Mytilus edulis, Evolution 30 (1976) 2-32. 
[20] Koornneef M., Alonso-Blanco C., Peeters A.J.M., Soppe W., Genetic control of flowering time in Arabidopsis thaliana, Annu. Rev. Plant Phys. 49 (1998) 345370.

[21] Le Boulc'h V., Évolution de la résistance à l'oïdium (Erysiphe graminis) dans des populations de blé tendre (Triticum aestivum L.) menées en gestion dynamique, Thesis, Institut national agronomique Paris-Grignon, Paris, 1994.

[22] Le Boulc'h V., David J.L., Brabant P., de Vallavieille-Pop C., Dynamic conservation of variability: responses of wheat populations to different selective forces including powdery mildew, Genet. Select. Evol. 26 (1994) 221s-240s.

[23] Levins R., Evolution in changing environments, Princeton University Press, Princeton, 1971.

[24] Martinez-Zapater J.M., Coupland G., Dean C., Koornneef M., The transition to flowering in Arabidopsis, in: Meyerowitz, M.E. and Somerville, C.R. (eds.), Arabidopsis, Cold Spring Harbor, New York, 1994, pp. 403-433.

[25] McCauley D.E., Evolution in metapopulations with frequent local extinction and recolonization, Oxford Surv. Evol. Biol. 10 (1993) 109-134.

[26] Mitchell-Olds T., Genetic constraints on life-history evolution: Quantitativetrait loci influencing growth and flowering in Arabidopsis thaliana, Evolution 50 (1996) 140-145.

[27] Mitton J.B., Koehn R.K., Genetic organization and adaptive response of allozymes to ecological variables in Fundulus heteroclitus, Genetics 79 (1975) 97111.

[28] Nei M., Molecular Evolutionary Genetics, Columbia University Press, New York, 1987.

[29] Ornstein P., Disc electrophoresis. I. Background and theory, Ann. N.Y. Acad. Sci. (1964) 321-347.

[30] Paillard S., Goldringer I., Enjalbert J., Doussinault G., de Vallavieille-Pope C., Brabant P., Evolution of resistance against powdery mildew in winter wheat populations conducted under dynamic management. I. Is specific seedling resistance selected? Theor. Appl. Genet. 101 (2000) 449-456.

[31] Paillard S., Goldringer I., Enjalbert J., Trottet M., David J., de Vallavieille-Pope C., Brabant P., Evolution of resistance against powdery mildew in winter wheat populations conducted under dynamic management. II. Adult plant resistance, Theor. Appl. Genet. 101 (2000) 457-462.

[32] Pasteur N., Pasteur G., Bonhomme F., Catalan J., Brutton-Davidian J., Manuel technique de génétique par électrophorèse de protéines, Lavoisier Tec. et Doc., Paris, 1987.

[33] Pernès J., Gestion des ressources génétiques des plantes, Lavoisier, 1984.

[34] Pigliucci M., Ecological and evolutionary genetics of Arabidopsis, Trends Plant Sci. 3 (1998) 485-489.

[35] Ruiz-Garcia L., Madueño F., Wilkinson M., Haughn G., Salinas J., MartinezZapater J.M., Different roles of flowering-time genes in the activation of floral initiation genes in Arabidopsis, Plant Cell 9 (1994) 1921-1934.

[36] Rumball W., Franklin I., Frankham R., Sheldon B., Decline in heterozygosity under full-sib and double-first cousin inbreeding in Drosophila melanogaster, Genetics 136 (1994) 1039-1049.

[37] Singh S.M., Zouris E., Genetic variation associated with growth rate in the American oyster (Crassostrea virginica), Evolution 32 (1978) 342-353. 
[38] Slatkin M., Wade M.J., Group selection on a quantitative character, Proc. Nat. Acad. Sci. USA 75 (1978) 3531-3534.

[39] Snape J.W., Lawrence M.J., The breeding system of Arabidopsis thaliana, Heredity 27 (1971) 299-302.

[40] Soltis D.E., Soltis P.S., Isozymes in plant biology, London, 1989.

[41] Tigerstedt P.M.A., Rudin D., Niemela T., Tammisola J., Competition and neighbouring effect in a naturally regenerating population of Scots pine, Silva Fenica 16 (1982) 122-129.

[42] Tracey M.L., Bellet N.F., Graven C.B., Excess of allozyme homozygosity and breeding population structure in the mussel Mytilus californianus, Mar. Biol. 32 (1975) 303-311.

[43] Wade M.J., McCauley D.E., Extinction and recolonization: their effects on the genetic differentiation of local populations, Evolution 42 (1988) 995-1005.

[44] Watt W.B., Donohue K., Carter P.A., Adaptation at specific loci. 6. Divergence vs parallelism of polymorphic allozymes in molecular function and fitnesscomponent effects among Colias species (Lepidoptera, Pieridae), Mol. Biol. Evol. 13 (1996) 699-709.

[45] Weir B.S., Cockerham C.C., Estimating F-statistics for the analysis of population structure, Evolution 38 (1984) 1358-1370.

[46] Whitlock M.C., McCauley D.E., Some population genetic consequences of colony formation and extinction: genetic correlations within founding groups, Evolution 44 (1990) 1717-1724.

[47] Zhang J., Lechowicz M.J., Correlation between time of flowering and phenotypic plasticity in Arabidopsis thaliana (Brassicaceae), Am. J. Bot. 81 (1994) 13361342. 